\title{
ABOVEGROUND BIOMASS ESTIMATION IN TROPICAL FORESTS AT SINGLE TREE LEVEL WITH ALS DATA
}

\author{
Michele Dalponte ${ }^{1,2}$, Tommaso Jucker ${ }^{2}$, David F. R. P. Burslem ${ }^{3}$, Simon L. Lewis ${ }^{4}$, Reuben Nilus ${ }^{5}$, \\ Oliver Phillips ${ }^{4}$, Lan $_{\text {Qie }}{ }^{4}$, and David A. Coomes ${ }^{2}$ \\ ${ }^{1}$ Department of Sustainable Agro-ecosystems and Bioresources, Research and Innovation Centre, \\ Fondazione E. Mach, Via E. Mach 1, 38010 San Michele all'Adige (TN), Italy. Email: \\ michele.dalponte@fmach.it \\ ${ }^{2}$ Forest Ecology and Conservation Group, Department of Plant Sciences, University of Cambridge, \\ Downing Street, Cambridge, CB2 3EA, UK. Email: tj272@cam.ac.uk; dac18@cam.ac.uk \\ ${ }^{3}$ The School of Biological Sciences, University of Aberdeen, Zoology Building, Tillydrone Avenue, \\ Aberdeen AB24 2TZ, UK. Email: d.burslem@abdn.ac.uk \\ ${ }^{4}$ School of Geography, University of Leeds, Leeds LS2 9JT, UK. Email: s.l.lewis@leeds.ac.uk; \\ o.phillips@leeds.ac.uk; 1.qie@leeds.ac.uk \\ ${ }^{5}$ Forest Research Centre, Sabah Forestry Department, P.O. Box 1407, 90715 Sandakan \\ Sabah, Malaysia. Email: reuben.nilus@ sabah.gov.my
}

\begin{abstract}
In this paper we present a study on the estimation of the aboveground biomass in tropical forests at single tree level using airborne laser scanning (ALS) data. Individual tree crowns (ITCs) are firstly detected using a method based on an adaptive window that change its size according to tree height. The diameter at breast height (DBH) and the aboveground biomass (AGB) of each ITC then are predicted using standard allometric models. Lastly, the AGB values are aggregated at plot level, and compared with field measured values. The results show that it is possible to accurately predict the aboveground biomass of tropical forests at single tree level using ALS data.
\end{abstract}

Index Terms - aboveground biomass, LiDAR, ALS, tropical forest.

\section{INTRODUCTION}

Forests are a major component in the global carbon cycle, and accurate estimation of forest carbon stocks and fluxes is important in the context of global warming [1]. Recently great attention has been devoted to the estimation of carbon stored by tropical forests inside the UN REDD program; on the one hand very little is known about the carbon stored by tropical forests, and on the other hand these forests are the most endangered by forest degradation and deforestation.

Airborne laser scanning (ALS) data are increasingly recognized as an outstanding data source for high-fidelity mapping of carbon stocks at regional scales [2]-[4]. ALS modelling has focused on developing regression relationships between estimates of carbon density (obtained from forest inventory plots) with plot-level summary statistics obtained from the ALS point clouds, and then applying the regression relationships to estimate carbon stocks across laser-scanned landscapes [5].

Recently in the literature many algorithms for individual tree crowns (ITC) detection have been presented (e.g. [6], [7]) leading to the possibility to have ITC level mapping of forest volume, aboveground biomass (AGB) and carbon. The advantages of working at the individual tree level instead of plots include that: i) the approach is fundamentally identical as that used in ground-based inventories, and is cleaner from a "theoretical" perspective; ii) a higher spatial detail can be obtained; and iii) the information obtained is completely scalable, both spatially (it can be aggregated over areas of different areas and shape) and by other attributes (e.g. species). Moreover working with individual tree crowns provides information that can be used for many other applications.

Up until now almost all studies that have estimated AGB using ITC approaches have been carried out on temperate and boreal forest, and very few studies have focused on the tropics. In order to fill this gap in the literature, we present a study on the prediction of AGB at single tree level carried on a tropical forest in Malaysia, using a new ITC delineation method. Beyond the fact that the use of ITC-level AGB prediction in tropical forests is a novelty in itself, another novelty of this work is the delineation method which optimizes its parameters according to forest characteristics. 


\section{DATA SET DESCRIPTION}

The study area is the Sepilok Natural Reserve located in the Sabah state of Malaysia. The area is characterized by a very high species richness (i.e. hundreds of tree species per hectare). The terrain is relatively flat and is characterized by three distinct soil types which give rise to very different forest types (hereafter alluvial, sandstone and kerangas; see Figure 1).

The field data are characterized by nine squared plots of 4 hectares. Three plots are located on alluvial soil, three on kerangas, and three on sandstone. Inside each plot all trees $>5 \mathrm{~cm}$ in diameter (DBH) have been measured, identified to species, and their position relative to subplots of 10 by $10 \mathrm{~m}$ recorded. For a subset of trees, tree height $(\mathrm{H})$ was also measured using a laser range finder. In the data analysis the nine 4 ha plots have been divided into 36 subplots of 1 hectare dimension.

ALS data covering the study area were acquired on the $5^{\text {th }}$ of November 2014 using a Leica ALS50-II sensor. Up to 4 returns per pulse have been acquired with a point density higher than $10 \mathrm{pts} / \mathrm{m}^{2}$.

\section{METHODS}

\subsection{ALS data preprocessing}

The raw ALS data were preprocessed using the software LAStools. In particular the ALS pulses have been classified in ground and non-ground points, and the relative $\mathrm{Z}$ for each ALS point has been computed.

\subsection{ITC delineation}

The automatic ITC delineation approach finds local maxima within a rasterized CHM, designates these as tree tops, then uses a decision tree method to grow individual crowns around the local maxima. The approach goes through the following steps: (1) a low-pass filter is applied to the rasterized CHM to smooth the surface and reduce the number of local maxima; (2) local maxima are located using a circular moving window with size that adapts inside a user defined range (minimum and maximum size) according the pixel height; a pixel of the CHM is labelled as local maxima if its $\mathrm{z}$ value is greater than all other $\mathrm{z}$ values in the window, and with $\mathrm{z}$ greater than some minimum height aboveground; (3) each local maximum is labelled as an "initial region" around which a tree crown can grow; the heights of the four neighboring pixels are extracted from the CHM and these pixels are added to the region if their vertical distance from the local maximum is less than some user-defined percentage of the local-maximum height, and less than some user-defined maximum difference; this procedure is repeated for all the neighbors of cells now included in the region, and so on iteratively until no further pixels are added to the region; (4) from each region that had been identified the first-return ALS points are extracted (having first removed low elevation points), (5) a $2 \mathrm{D}$ convex hull is applied to these points, and the resulting polygons becomes
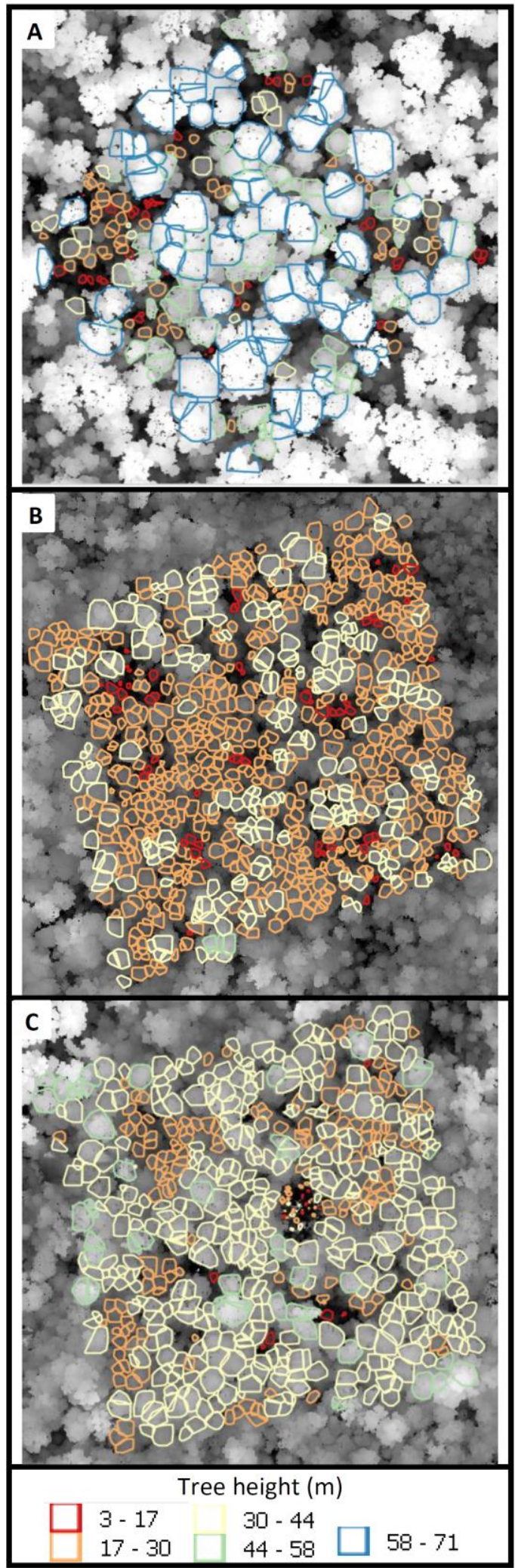

Figure 1. Canopy Height Model of three field measured plots (plot $\mathrm{A}$ refers to an alluvial soil, $\mathrm{B}$ to kerangas and $\mathrm{C}$ to sandstone) with overlaid the ITCs delineated using the optimized window sizes. 
the final ITCs. This delineation approach is implemented in the R library itcSegment.
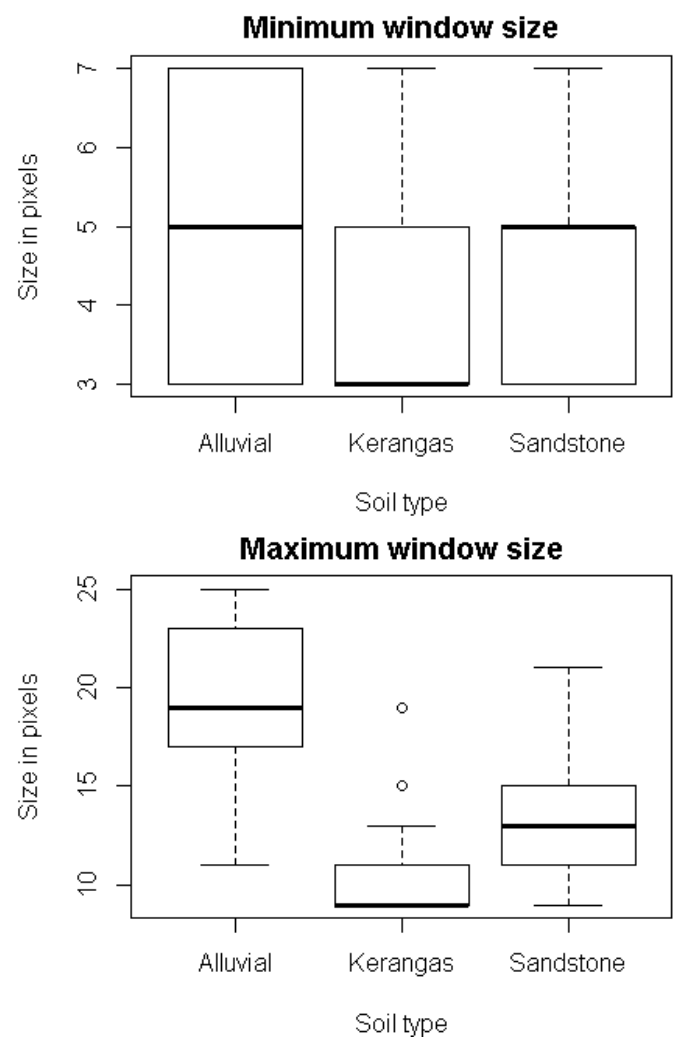

Figure 2. Searching windows sizes versus soil type.

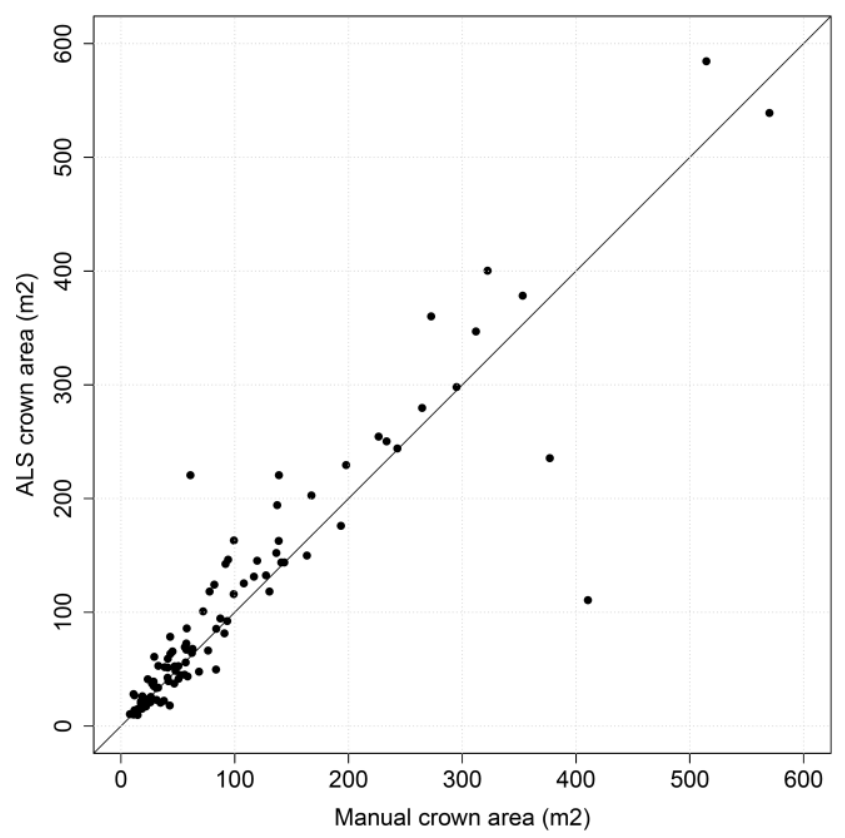

Figure 3. Crown area of the manually delineated ITCs versus crown area of the ALS delineated ITCs.

\subsection{DBH and AGB estimation}

The DBH for the detected ITCs was computed using allometric models developed starting from the field data (i.e. field measured height and diameter) collected in the study area. In particular three diameter-height models have been developed, one for each soil type, in the form: $D B H=a *$ $H^{b}$. As height $(\mathrm{H})$ we used the maximum height of the ALS points inside each detected ITC.

The AGB of each ITC was computed using the formulas of Chave et al. [8]:

$$
A G B=0.0673 *\left(\rho * D B H^{2} * H\right)^{0.976}
$$

where $\rho$ is the wood density. A different value of $\rho$ for each soil type was used.

\subsection{Sensitivity analysis and accuracy assessment}

The results of the ITC delineation method used in this study are mainly driven by two parameters: the minimum and maximum size of the moving window used to locate the local maxima. A sensitivity analysis was carried out to test their effect on the AGB estimation at plot level. In particular we tested minimum window sizes from 3 to 7 , and maximum window sizes from 9 to 25 . For each combination ITCs were delineated over the 1 ha subplots and DBH and AGB were predicted for each tree. AGBs were than aggregated at subplot level (1 ha plots) and the difference between ALS estimated and field estimated AGB for each plot were computed. The set of window sizes giving the smallest difference in each plot was chosen as the optimal for each plot.

The delineation method was also validated on a set of 147 manually delineated ITCs. In particular in the validation we considered the detection rate (DET) and omission error $(\mathrm{OE})$. Moreover a comparison among the area of the ALS and manually delineated ITCs was done.

The manually delineated ITCs were used also to build up three models (one for each soil type) for the prediction of the crown diameter using the tree height. Crown dimeter predicted with these models was used to set the maximum window size of the automatic delineation method.

\section{RESULTS}

The soil type has strong influence on the forest structure, height and composition. This is showed by the sensitivity analysis results. From Figure 1 it is clear that, especially for the maximum window size, there is a great difference among the soils. The forest located on kerangas soil is characterized by smaller trees and thus a smaller window size is needed. Differently on alluvial soil we have very big emerging trees and thus, in the plots in which these trees are present ,a bigger window size is needed. On the basis of these results we kept the minimum window size fixed at 3 , and we adapted the maximum window size in each 1 ha 
subplot. In particular the maximum window size was fixed using the crown dimeter-height models derived from the manually delineated ITCs.

The detection rate of the automatically delineated ITCs versus the manual ones was $71.4 \%$. The commission error was $28.6 \%$. As it is possible to see from Figure 3 there is a good agreement among the crown areas for the detected ITCs $\left(\mathrm{R}^{2}\right.$ of 0.85$)$. It is worth noting that manually delineated tree crowns were also delineated on ALS data. In Figure 1 it is possible to see an example of the delineated ITCs. Analyzing visually the plots it seems that there is a good matching between the delineated ITCs and the real crowns.

Figure 4 shows the results in terms of AGB at plot level. The ALS-estimated AGB was corrected with a correction factor of 1.39 like in [9]. The $\mathrm{R}^{2} 0.58$ and the nRMSE (\%) is $15.6 \%$.

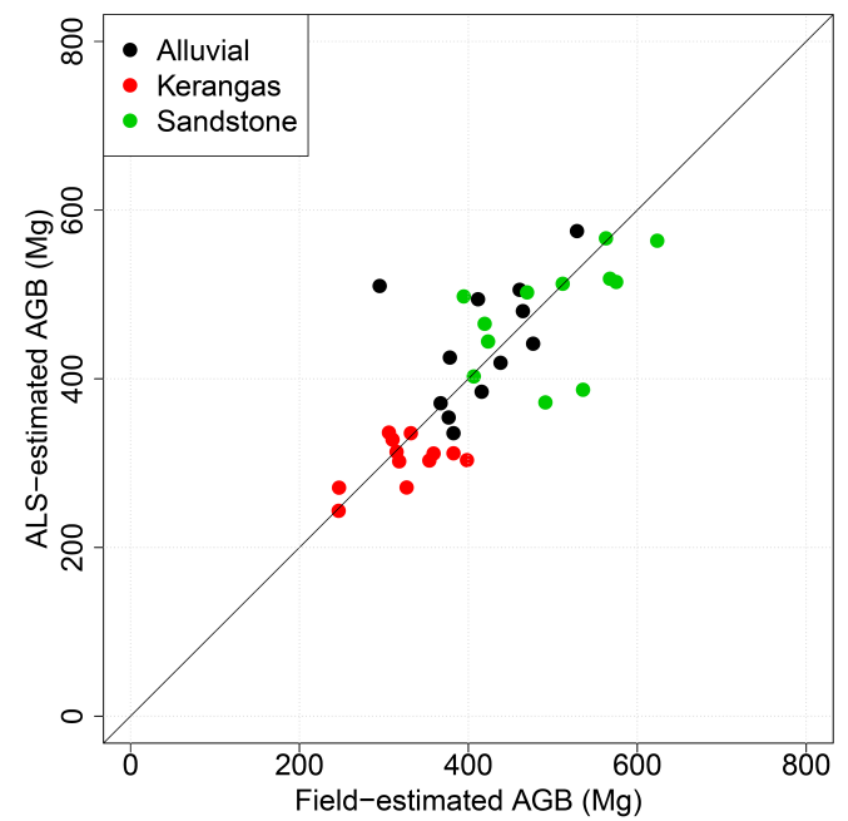

Figure 3. Field-estimated AGB and ALS-estimated AGB for each 1 ha subplot.

\section{CONCLUSIONS}

In this paper we showed that the estimation of aboveground biomass at ITC level in tropical forests is possible, and the results obtained are promising for future research. Some limitation remains connected to the fact that not all the trees can be detected by the ITCs delineation methods available, and this is creating a bias in the estimation that we bypassed using a correction factor. Future research should focus on better understanding how to have an a priori knowledge of this correction factor in order to solve this problem.

\section{ACKNOWLEDGEMENTS}

The research leading to these results was supported by the Biodiversity and Land Use Intensity (BALI) project financed by Natural Environment Research Council (NERC).

\section{REFERENCES}

[1] Y. Pan, R. a. Birdsey, O. L. Phillips, and R. B. Jackson, "The Structure, Distribution, and Biomass of the World's Forests," Annu. Rev. Ecol. Evol. Syst., vol. 44, no. 1, pp. 593-622, 2013.

[2] G. P. Asner, J. Mascaro, H. C. Muller-Landau, G. Vieilledent, R. Vaudry, M. Rasamoelina, J. S. Hall, and M. van Breugel, "A universal airborne LiDAR approach for tropical forest carbon mapping," Oecologia, vol. 168, no. 4, pp. 1147-1160, Apr. 2012.

[3] E. H. Hansen, T. Gobakken, and E. Næsset, "Effects of pulse density on digital terrain models and canopy metrics using airborne laser scanning in a tropical rainforest," Remote Sens., vol. 7, no. 7, pp. 8453-8468, 2015.

[4] G. P. Asner and J. Mascaro, "Mapping tropical forest carbon: Calibrating plot estimates to a simple LiDAR metric," Remote Sens. Environ., vol. 140, pp. 614-624, 2014.

[5] E. Næsset, "Predicting forest stand characteristics with airborne scanning laser using a practical two-stage procedure and field data," Remote Sens. Environ., vol. 80, no. 1, pp. 88-99, Apr. 2002.

[6] J. Vauhkonen, L. Ene, S. Gupta, J. Heinzel, J. Holmgren, J. Pitkänen, S. Solberg, Y. Wang, H. Weinacker, K. M. Hauglin, V. Lien, P. Packalén, T. Gobakken, B. Koch, E. Næsset, T. Tokola, and M. Maltamo, "Comparative testing of single-tree detection algorithms under different types of forest," Forestry, vol. 85, no. 1, pp. 27-40, Oct. 2012.

[7] L. Eysn, M. Hollaus, E. Lindberg, F. Berger, J.-M. Monnet, M. Dalponte, M. Kobal, M. Pellegrini, E. Lingua, D. Mongus, and N. Pfeifer, "A Benchmark of Lidar-Based Single Tree Detection Methods Using Heterogeneous Forest Data from the Alpine Space," Forests, vol. 6, no. 5, pp. 1721-1747, 2015.

[8] J. Chave, M. Réjou-Méchain, A. Búrquez, E. Chidumayo, M. S. Colgan, W. B. C. Delitti, A. Duque, T. Eid, P. M. Fearnside, R. C. Goodman, M. Henry, A. Martínez-Yrízar, W. a. Mugasha, H. C. Muller-Landau, M. Mencuccini, B. W. Nelson, A. Ngomanda, E. M. Nogueira, E. Ortiz-Malavassi, R. Pélissier, P. Ploton, C. M. Ryan, J. G. Saldarriaga, and G. Vieilledent, "Improved allometric models to estimate the aboveground biomass of tropical trees," Glob. Chang. Biol., vol. 20, pp. 3177-3190, 2014.

[9] M. Dalponte and D. A. Coomes, "Tree-centric mapping of forest carbon density from airborne laser scanning and hyperspectral data," Methods Ecol. Evol., 2016. 